Case Report

\title{
Safety and Efficacy of Triple Therapeutic Targets with Rivaroxaban after Acute Myocardial Infarction Complicated by Left Ventricular Thrombi in a Case of Nonvalvular Atrial Fibrillation
}

\author{
Francesco Summaria (D), Gregory A. Sgueglia, Fabrizio D'Errico, Antonella De Santis, \\ Fabiana Piccioni, Gaetano Gioffrè, and Achille Gaspardone
}

Department of Cardiology, San Eugenio Hospital, Rome, Italy

Correspondence should be addressed to Francesco Summaria; f.summaria@gmail.com

Received 5 December 2017; Accepted 14 January 2018; Published 5 March 2018

Academic Editor: Nurten Sayar

Copyright $(\odot) 2018$ Francesco Summaria et al. This is an open access article distributed under the Creative Commons Attribution License, which permits unrestricted use, distribution, and reproduction in any medium, provided the original work is properly cited.

We present the complex case of a high-risk patient with nonvalvular atrial fibrillation, who experienced a non-ST elevation myocardial infarction complicated by left ventricular (LV) thrombi and underwent percutaneous coronary intervention with drug-eluting stent implantation. The patient was initially treated with short-term triple therapy including aspirin, clopidogrel, and rivaroxaban $15 \mathrm{mg} /$ die. Following aspirin dropping one month after discharge, the patient continued on dual therapy with clopidogrel and rivaroxaban, and a clinical and imaging follow-up at 6 and 12 months confirmed the LV thrombi resolution, with no thromboembolic episodes and a good safety profile.

\section{Introduction}

Among acute coronary syndrome (ACS) patients undergoing percutaneous coronary intervention (PCI), approximately $5 \%$ to $22 \%$ have concomitant atrial fibrillation (AF) [1]. Among ACSs, AF is 2-fold more frequent during non-ST elevation myocardial infarction (NSTEMI) than during STEMI [2]. Despite the overlap in the occurrence of these syndromes, the pharmacotherapies used to manage AF and ACSs differ.

Left ventricular (LV) thrombi often represent an unexpected echocardiographic finding [3], accurately detected by cardiovascular magnetic resonance (CMR) [4]. Although the occurrence of this complication has declined significantly since the advent of primary PCI, its incidence is not negligible, ranging between $5 \%$ and $17 \%[5,6]$.

According to current guidelines, despite an increased rate of bleeding complications, warfarin is considered the anticoagulant of choice to prevent thromboembolic sequelae. As an advantageous alternative, the novel oral anticoagulants (NOACs) have been implemented in the setting of nonvalvular AF
(NVAF) [7-9]. Yet, the evidence about their role in combination with antiplatelet agents in the management of LV thrombi for ACS patients undergoing PCI is poor, and mostly limited to case reports. Hence, their efficacy can be only extrapolated [10-13]. At present, two randomized trials (PIONEER AF-PCI and REDual PCI) $[14,15]$ have demonstrated that the use of NOACs plus a P2Y12 inhibitor was associated with a lower rate of clinically significant bleeding, compared to VKAs plus dual antiplatelet therapy, thus ensuring a dual target of ischemic and thromboembolic protection against coronary and cerebrovascular events.

\section{Case Report}

We report the case of a 66-year-old woman, hypertensive, and smoker, referred to our Emergency Department for prolonged chest pain. Due to permanent NVAF, she was on warfarin and rate-control therapeutic strategy. At admission, electrocardiogram showed AF and a ST segment depression of $2 \mathrm{~mm}$ in V5-V6. The creatine kinase-MB was of 10 and 


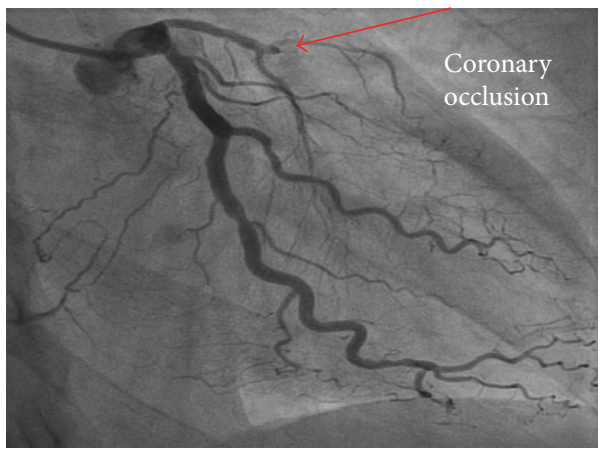

(a)

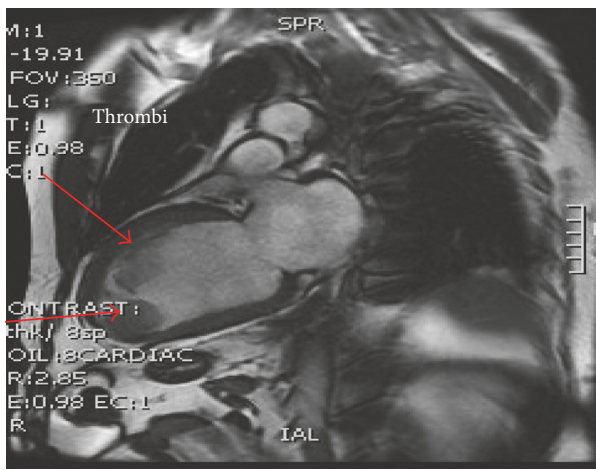

(c)

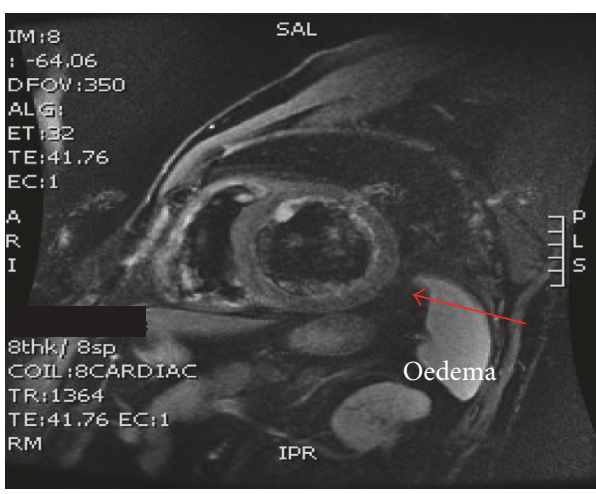

(e)

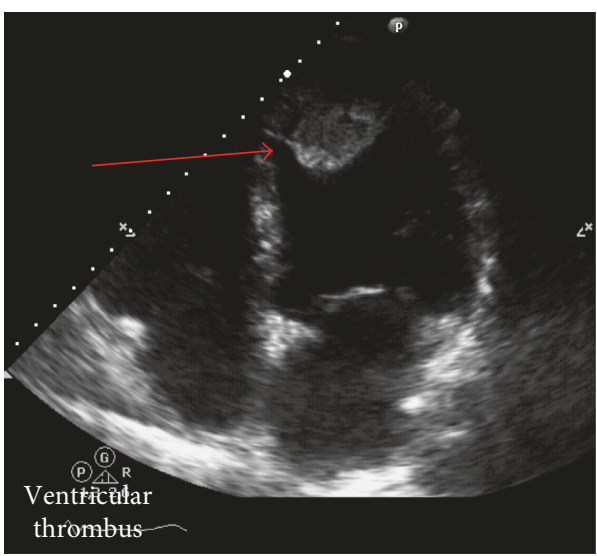

(b)

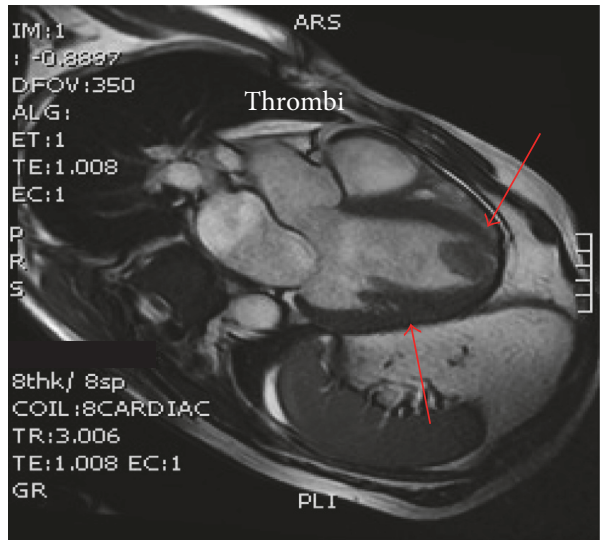

(d)

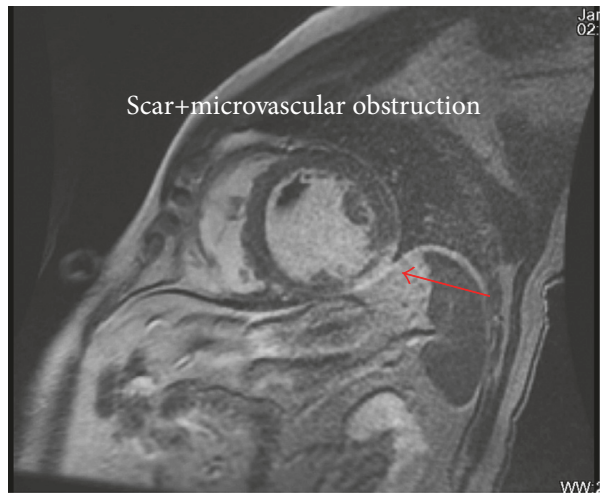

(f)

Figure 1: Continued. 


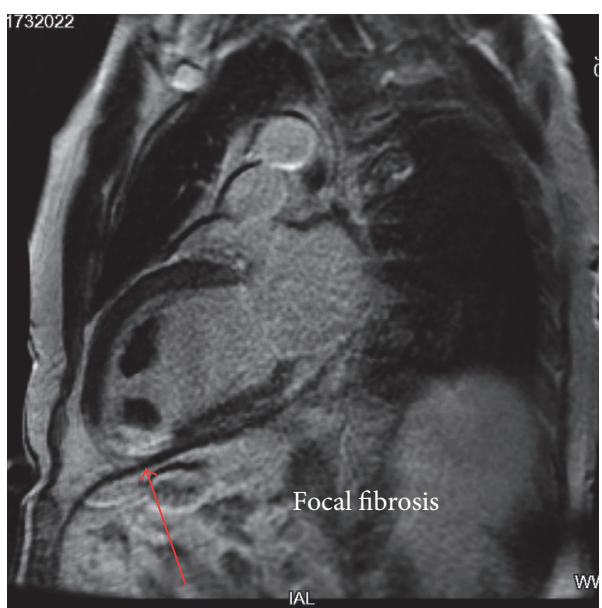

(g)

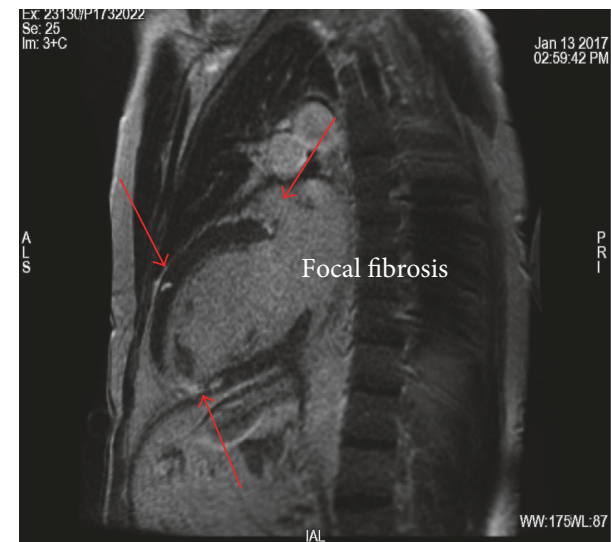

(h)

FIGURE 1: (a) Coronary angiography showing the occlusion of the left descending artery in the proximal segment (arrow); (b) transthoracic 2-D echocardiography showing an apical ventricular thrombus; (c, d) cardiac magnetic resonance (CMR) unveiling the presence of two large left ventricular thrombi in the apex and along the anterior wall; (e) CMR TIR-T2 sequences showing myocardial edema involving the anterior wall of the left ventricle; (f, g) delay enhancement revealing scar, microvascular obstruction, and fibrosis (arrows); (h) CMR imaging: three hyperenhancement focal areas of fibrosis.

high-sensitivity troponin I was of $6.0 \mathrm{ng} / \mathrm{mL}$, with normal hemoglobin level and INR of 1.9. At baseline, a combined thromboembolic and bleeding scoring system evaluation was performed, resulting in a $\mathrm{CHA}_{2} \mathrm{DS}_{2}$-VASc of 4 and HAS-BLED of 3 [16-18]. A NSTEMI diagnosis was made, and an antiplatelet therapy with aspirin $300 \mathrm{mg}$, clopidogrel $600 \mathrm{mg}$ loading dose, and intravenous unfractionated heparin $5000 \mathrm{IU}$ was administered, while an early invasive strategy with a transradial approach was planned. The coronary angiography showed a normal right coronary artery and the occlusion of the left descending artery in the proximal segment (Figure 1(a)). An IVUS-guided PCI was performed, with the implantation of two overlapped last-generation DES (Xience Alpine, Abbott), $2.75 \times 28 \mathrm{~mm}$ and $3.0 \times 28 \mathrm{~mm}$, respectively, both expanded up to 16 atmospheres. In order to optimize expansion and avoid malapposition, the stents were overexpanded, with noncompliant balloons of $3.5 \times 15 \mathrm{~mm}$ (NC Quantum, Boston), up to 20 atmospheres, according to IVUS-guided vessel sizing.

Considering the clinical setting (NSTEMI $+\mathrm{AF}$ ) and the procedure (PCI with DES implantation), the antiplatelet therapy with aspirin $100 \mathrm{mg}$ /day plus clopidogrel $75 \mathrm{mg}$ /day was continued and anticoagulation with rivaroxaban $15 \mathrm{mg} /$ day was started immediately after PCI. The transthoracic 2-D echocardiography revealed a hypokinesia of the apex and anterior wall with moderate reduction of the ejection fraction. Two mobile masses were found in the apex and in the anterior wall of the left ventricle, respectively (Figure 1(b)). A CMR was performed at day three, confirming apex and anterior wall hypokinesia and $40 \%$ ejection fraction. CMR unveiled the presence of two LV thrombi in the apex and along the anterior wall, respectively (Figures 1(c) and 1(d)). TIR-T2 sequences showed myocardial oedema (Figure 1(e)) involving the LV anterior wall; delay enhancement (Figures $1(\mathrm{f})$ and $1(\mathrm{~g})$ ) revealed a thickness fibrosis in the same segment, with a hypoenhanced internal area suggesting microvascular obstruction. Three hyperenhancement focal areas were detected (Figure 1(h)): a transmural area at inferior apical septum, just beneath one thrombus, and two subepicardial areas, at anterior basal septum and at midanterior wall, respectively.

The patient was discharged 7 days after admission. A clinical and cardiovascular imaging follow-up was planned at 1 month, 6 months, and 1 year. According to current guidelines and expert consensus knowledge $[19,20]$, the antithrombotic/anticoagulant therapy was managed as follows: at 1 month, the thromboembolic and bleeding risk scores $\left(\mathrm{CHA}_{2} \mathrm{DS}_{2}\right.$-VASc of 4 and HAS-BLED of 3) were rechecked, the antiplatelet therapy with clopidogrel together with the anticoagulant therapy with rivaroxaban $15 \mathrm{mg} /$ day was confirmed, and aspirin was stopped considering the high bleeding risk. At 6 months, no ischemic thromboembolic or bleeding events were reported. The echo and CMR imaging confirmed the resolution of the LV thrombi. Despite the fact that MRI are not necessary to confirm the thrombi resolution documented by echocardiogram, in our institution CMR facility together with an internal protocol in the case of a newer therapeutic strategy led us to perform this modality of cardiac imaging for speculative purpose. The dual therapy with clopidogrel plus rivaroxaban was confirmed [14].

At 1 year, a clinical follow-up confirmed the good clinical results: the patient was asymptomatic, and neither ischemic coronary events nor thromboembolic or bleeding events were reported. After 1 year, considering the $\mathrm{CHA}_{2} \mathrm{DS}_{2}$-VASc score, the high rate of recurrence of events after NSTEMI, the use of DES, the absence of bleeding events during previously dual antiplatelet therapy, a treatment with rivaroxaban plus aspirin was confirmed. 


\section{Discussion}

The optimal antithrombotic strategy for patients with NVAF and ACS undergoing PCI is still controversial, and the role of NOACs in this setting is poorly documented [10-14].

The guidelines of ACS and AF report how to manage the antiplatelet and anticoagulant therapies in overlapped situations, but, frequently, the indication and level of evidence are strong only when the conditions are considered alone $[19,20]$. Many different combinations have been proposed based on the use of different agents and for varying duration, but as the final target must balance the risk of bleeding and ischemic events in each patient, it is difficult to make a definitive decision especially when other potential thromboembolic complications (e.g., LV thrombi) occur. NOACs are at least as effective as warfarin in terms of thromboembolic complications, but safer in terms of bleeding complications $[8,9]$. As for the dose, in the dual or triple therapy, guidelines recommend the lower available dose of NOACs $[19,20]$. Based on the recent results of the PIONEER AF-PCI trial [14], the more effective and safer ratio is clopidogrel $75 \mathrm{mg}$ plus rivaroxaban $15 \mathrm{mg}$, or either clopidogrel or ticagrelor plus dabigatran at $110 \mathrm{mg}$ or $150 \mathrm{mg}$ (RE-DUAL PCI) [21]. In contrast with the currently available data in which the evidence of the efficacy and safety of NOACs in patients with ACS and AF undergoing PCI is limited to a low HASBLED score, our patient had a high bleeding risk; therefore, despite this acute setting, a triple therapy was used for 4 weeks only, followed by dual therapy with clopidogrel plus rivaroxaban $15 \mathrm{mg} /$ die for 12 months [14].

The use of aspirin has been reconsidered in the subgroup of patients at high risk of bleeding: for 6 months only in case of low bleeding risk, while for one month in case of high bleeding risk $[19,20]$. However, given the results of PIONEER and RE-Dual PCI, it is likely that aspirin will be definitively abandoned.

Periprocedural and technical cautions are crucial to minimize either the risk of access site bleeding or the risk of stent thrombosis and recurrence of coronary events. The transradial approach is mandatory also because it shortens the time to restart oral anticoagulation. In our case, the use of the latest-generation fluoro-passivated everolimus-eluting stent, as recently documented [22], lowers the rate of stent thrombosis and thrombotic complications. Intracoronary imaging with an IVUS-guided stent implantation reduces vessel wall malapposition and, consequently, stent thrombosis, thus improving a tailored pharmacological management.

Our combined interventional and pharmacological strategy aimed at balancing the ischemic and bleeding risk was also based on the use of the lower dose of NOAC tested for stroke prevention, and clopidogrel instead of the more potent ticagrelor and prasugrel, as suggested by guidelines and expert consensus $[19,20]$.

Although coronary DES significantly reduced the recurrences during the follow-up, the likelihood of a new event is not negligible. In this context, the use of NOACS seems to be a safe and effective option when the patient complexity and frailty due to higher combined thromboembolic and bleeding risk require to achieve multiple therapeutic targets.

\section{Disclosure}

Bayer HealthCare was not involved in the collection, analysis, and interpretation of data. In case of acceptance for publication, Bayer HealthCare will pay the article processing fee.

\section{Conflicts of Interest}

The authors declare that they have no conflicts of interest regarding the publication of this article.

\section{Acknowledgments}

The authors would like to thank Clara Ricci, PhD (Primula Multimedia SRL, Pisa, Italy), who provided skillful editorial assistance. Financial support for editorial services was provided by Bayer HealthCare, Italy.

\section{References}

[1] A. Rubboli, M. Colletta, J. Valencia et al., "Periprocedural management and in-hospital outcome of patients with indication for oral anticoagulation undergoing coronary artery stenting," Journal of Interventional Cardiology, vol. 22, no. 4, pp. 390-397, 2009.

[2] L. De Luca, S. Leonardi, C. Cavallini et al., "Contemporary antithrombotic strategies in patients with acute coronary syndrome admitted to cardiac care units in Italy: the EYESHOT study," European Heart Journal: Acute Cardiovascular Care, vol. 4, no. 5, pp. 441-452, 2015.

[3] F. Chiarella, E. Santoro, S. Domenicucci, A. Maggioni, and C. Vecchio, "Predischarge two-dimensional echocardiographic evaluation of left ventricular thrombosis after acute myocardial infarction in the GISSI-3 study," American Journal of Cardiology, vol. 81, no. 7, pp. 822-827, 1998.

[4] C. Lanzillo, M. Di Roma, A. Sciahbasi et al., "Cardiac magnetic resonance detection of left ventricular thrombus in acute myocardial infarction," Acute Cardiac Care, vol. 15, no. 1, pp. 11-16, 2013.

[5] G. Pizzetti, G. Belotti, A. Margonato et al., "Thrombolytic therapy reduces the incidence of left ventricular thrombus after anterior myocardial infarction. Relationship to vessel patency and infarct size," European Heart Journal, vol. 17, no. 3, pp. 421-428, 1996.

[6] D. Nayak, W. S. Aronow, R. Sukhija, J. A. McClung, C. E. Monsen, and R. N. Belkin, "Comparison of frequency of left ventricular thrombi in patients with anterior wall versus non-anterior wall acute myocardial infarction treated with antithrombotic and antiplatelet therapy with or without coronary revascularization," American Journal of Cardiology, vol. 93, no. 12, pp. 1529-1530, 2004.

[7] H. Heidbuchel, P. Verhamme, M. Alings et al., "European Heart Rhythm Association, European Heart Rhythm Association Practical Guide on the use of new oral anticoagulants in patients with non-valvular atrial fibrillation," Europace Journal, vol. 15, no. 5, pp. 625-651, 2013.

[8] M. R. Patel, K. W. Mahaffey, J. Garg et al., "Rivaroxaban versus warfarin in nonvalvular atrial fibrillation," New England Journal of Medicine, vol. 365, no. 10, pp. 883-891, 2011.

[9] C. T. Ruff, R. P. Giugliano, E. Braunwald et al., "Comparison of the efficacy and safety of new oral anticoagulants with warfarin in patients with atrial fibrillation: a meta-analysis of 
randomised trials," The Lancet, vol. 383, no. 9921, pp. 955962, 2014.

[10] C. A. Makrides, "Resolution of left ventricular postinfarction thrombi in patients undergoing percutaneous coronary intervention using rivaroxaban in addition to dual antiplatelet therapy," BMJ Case Reports, vol. 2016, p. bcr2016217843, 2016.

[11] Y. Mano, K. Koide, H. Sukegawa, M. Kodaira, and T. Ohki, "Successful resolution of a left ventricular thrombus with apixaban treatment following acute myocardial infarction," Heart and Vessels, vol. 31, no. 1, pp. 118-123, 2016.

[12] R. Seecheran, V. Seecheran, S. Persad, and N. A. Seecheran, "Rivaroxaban as an antithrombotic agent in a patient with STsegment elevation myocardial infarction and left ventricular thrombus: a case report," Journal of Investigative Medicine High Impact Case Reports, vol. 5, no. 1, p. 2324709617697991, 2017.

[13] C. Hammerstingl, B. Pötzsch, and G. Nickenig, "Resolution of giant left atrial appendage thrombus with rivaroxaban," Thrombosis and Haemostasis, vol. 109, no. 4, pp. 583-584, 2013.

[14] C. M. Gibson, R. Mehran, C. Bode et al., "Prevention of bleeding in patients with atrial fibrillation undergoing PCI," New England Journal of Medicine, vol. 375, no. 25, pp. 24232434, 2016.

[15] C. P. Cannon, D. L. Bhatt, J. Oldgren et al., "Antithrombotic therapy with dabigatran after PCI in atrial fibrillation," New England Journal of Medicine, vol. 377, no. 16, pp. 1513-1524, 2017.

[16] G. Y. H. Lip, R. Nieuwlaat, R. Pisters, D. A. Lane, and H. J. G. M. Crijns, "Refining clinical risk stratification for predicting stroke and thromboembolism in atrial fibrillation using a novel risk factor-based approach: the euro heart survey on atrial fibrillation," Chest, vol. 137, no. 2, pp. 263272, 2010.

[17] G. Y. H. Lip, L. Frison, J. L. Halperin, and D. A. Lane, "Comparative validation of a novel risk score for predicting bleeding risk in anticoagulated patients with atrial fibrillation: the HAS-BLED (hypertension, abnormal renal/liver function, stroke, bleeding history or predisposition, labile inr, elderly, drugs/alcohol concomitantly) score," Journal of the American College of Cardiology, vol. 57, no. 2, pp. 173-180, 2011.

[18] S. Apostolakis, D. A. Lane, H. Buller, and G. Y. H. Lip, "Comparison of the CHADS2, CHA2DS2-VASc and HASBLED scores for the prediction of clinically relevant bleeding in anticoagulated patients with atrial fibrillation: the AMADEUS trial," Thrombosis and Haemostasis, vol. 110, no. 5, pp. 10741079, 2013.

[19] G. Y. H. Lip, S. Windecker, K. Huber et al., "Management of antithrombotic therapy in atrial fibrillation patients presenting with acute coronary syndrome and/or undergoing percutaneous coronary or valve interventions: a joint consensus document of the European Society of Cardiology Working Group on Thrombosis, European Heart Rhythm Association (EHRA), European Association of Percutaneous Cardiovascular Interventions (EAPCI) and European Association of Acute Cardiac Care (ACCA) endorsed by the Heart Rhythm Society (HRS) and Asia-Pacific Heart Rhythm Society (APHRS)," European Heart Journal, vol. 35, no. 45, pp. 3155-3179, 2014.

[20] M. Roffi, C. Patrono, J.-P. Collet et al., "2015 ESC guidelines for the management of acute coronary syndromes in patients presenting without persistent ST-segment elevation," European Heart Journal, vol. 37, no. 3, pp. 267-315, 2016.

[21] C. P. Cannon, S. Gropper, D. L. Bhatt et al., "Design and rationale of the RE-DUAL PCI trial: a prospective, randomized, phase $3 \mathrm{~b}$ study comparing the safety and efficacy of dual antithrombotic therapy with dabigatran etexilate versus warfarin triple therapy in patients with nonvalvular atrial fibrillation who have undergone percutaneous coronary intervention with stenting," Clinical Cardiology, vol. 39, no. 10, pp. 555-564, 2016.

[22] T. Palmerini, U. Benedetto, G. Biondi-Zoccai et al., "Longterm safety of drug-eluting and bare-metal stents: evidence from a comprehensive network meta-analysis," Journal of the American College of Cardiology, vol. 65, no. 23, pp. 2496-2507, 2015. 


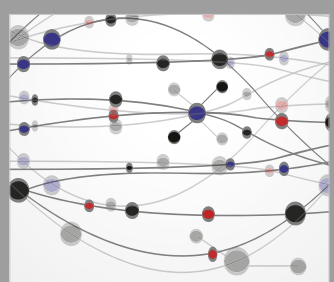

The Scientific World Journal
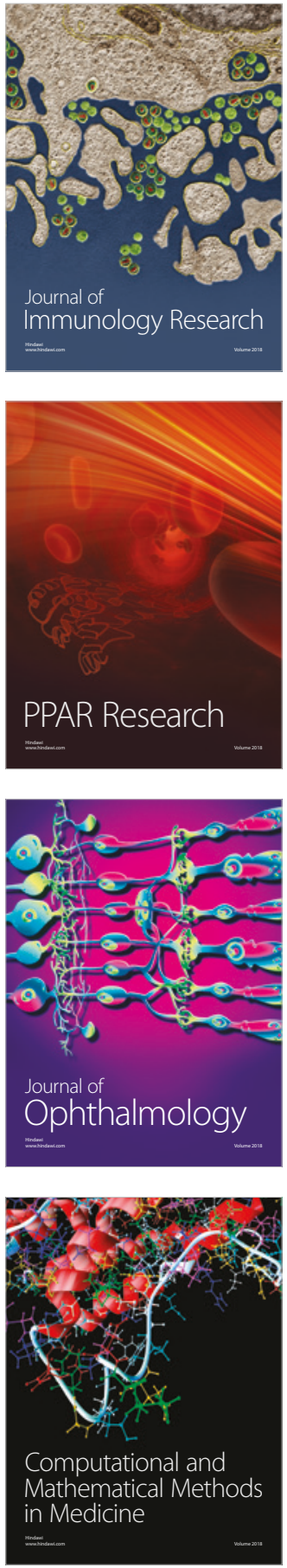

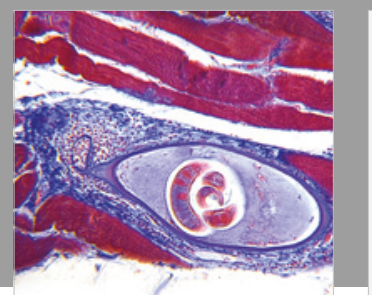

Gastroenterology Research and Practice

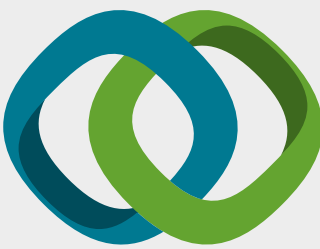

\section{Hindawi}

Submit your manuscripts at

www.hindawi.com
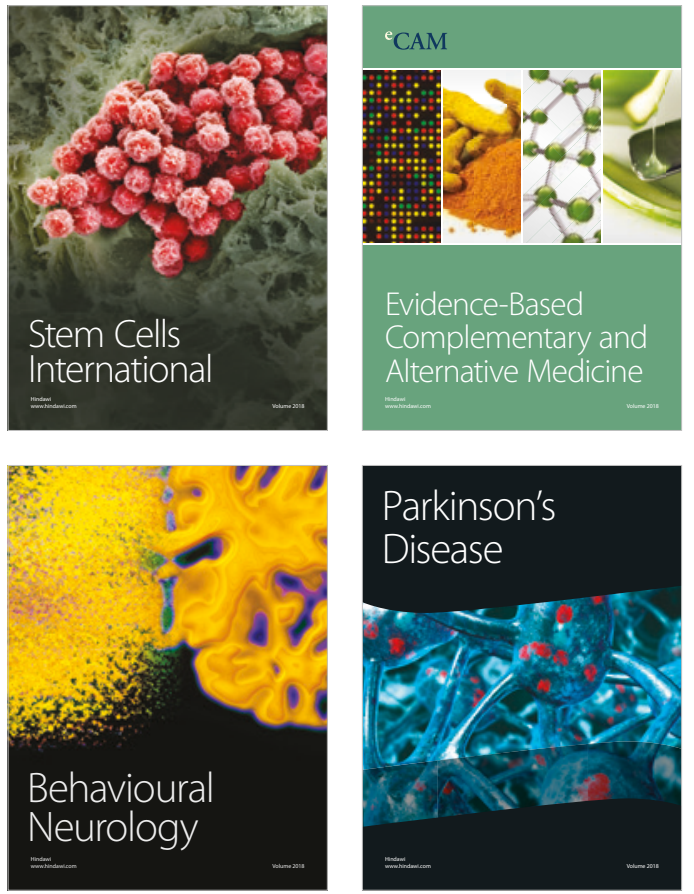

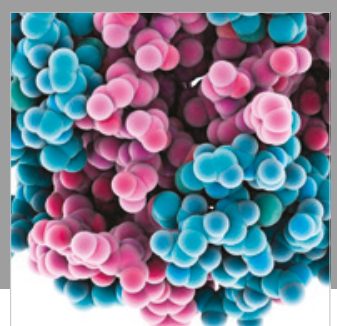

ournal of

Diabetes Research

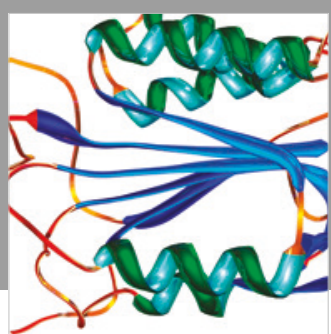

Disease Markers
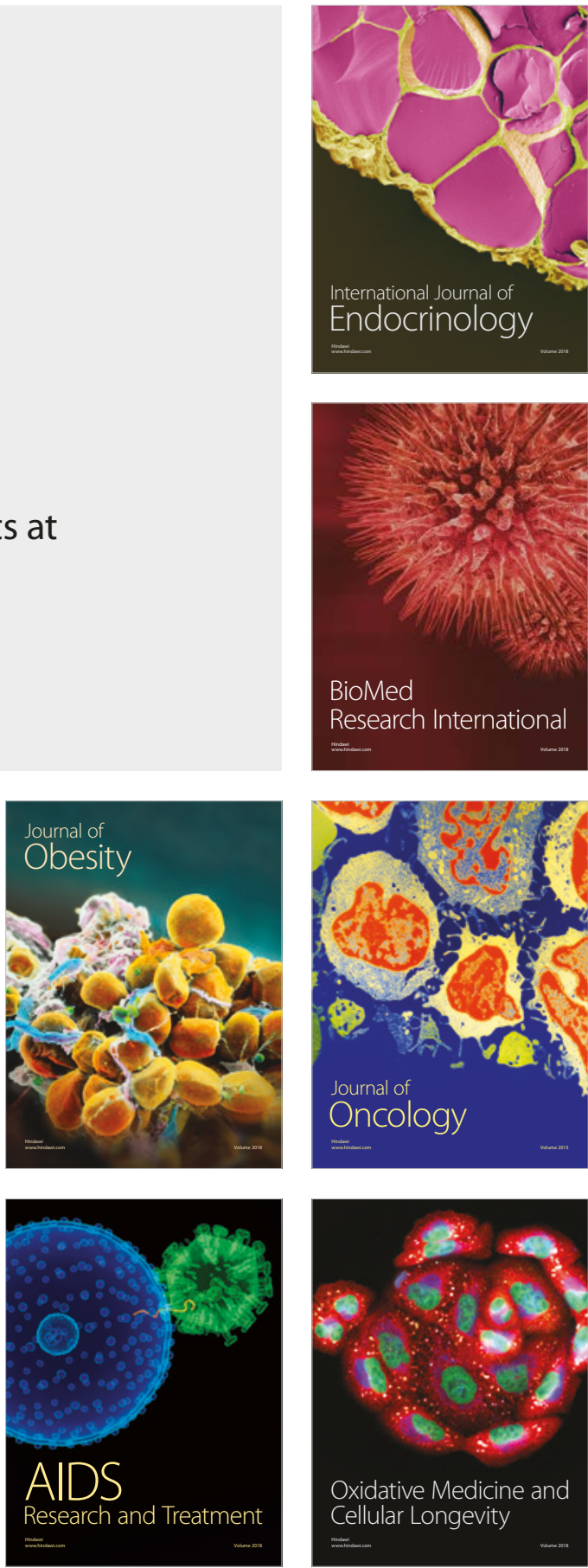